\title{
EFFECTS OF THE VARIATIONS IN THE CUMULATIVE LINER WALL THICKNESS ON THE PARAMETERS OF CUMULATIVE JET FORMATION PROCESS
}

\author{
Stancho P. Petkov, Petko S. Petkov, \\ ADELINA D. TUMBARSKA* \\ Acad. Angel Balevski Institute of Metal Science, Equipment and Technologies \\ with Hydro- and Aerodynamics Centre, Bulgarian Academy of Sciences, \\ 67, Shipchenski prohod St., 1574 Sofia, Bulgaria
}

[Received: 25 August 2019. Accepted: 03 February 2020]

doi: 10.7546/JTAM.50.20.02.06

\begin{abstract}
A review of the impact of various geometric, technological, and other imperfections in the shaped charges design on the parameters and characteristics of the cumulative jet formation process is made. A physicalmathematical model is proposed, on the basis of which numerical simulation is performed, allowing quantifying the influence of variations in the cumulative liner wall thickness on the deviation from the symmetry of the jet. The results of the calculations are presented in a graphical form, allowing for their use in further theoretical studies of the shaped charges functioning as well as in the design of ammunitions with predefined cumulative jet parameters.
\end{abstract}

KEY WORDS: shaped charge, cumulative jet formation, cumulative liner, wall thickness variation, cumulative jet symmetry.

\section{INTRODUCTION}

The effect of the cavity in explosives, discovered by chance in mining, has been subject of research for over 150 years. Its use to penetrate the armors of battle machines has generated interest among researchers in the early twentieth century, with major efforts being made to form axisymmetric, high-density, non-gradient jets to ensure high kinetic energy at impact [1] Until the middle of the century a significant development in the understanding the phenomena associated with cumulative jets has been made, further considerable advancement has been achieved in the next decades, as a result of the refinement of experimental methods and the use of high-speed video recording and radiography [2]. Contemporary applications of shaped charges, in addition to ammunition, are also related to a number of civilian sectors such as oil and gas production, mining, construction, demolition of buildings and massive structures, etc.

\footnotetext{
${ }^{*}$ Corresponding author e-mail: y.toumbarski@ims.bas.bg
} 
Currently, the presence of high-speed computers and powerful programs enable modeling and simulation of the processes that take place in the formation of the cumulative jet and its interaction with the barrier, which allow for these processes to be described quite accurately [3]. Despite the advances in this field, designing shaped charges with increasing efficiency remains a difficult engineering task that could be solved through considering the influence of a number of factors.

Shaped charged efficiency has been continuously increased in the recent years, but on the other hand, methods and materials for armor protection have also undergone major changes in order to meet this challenge. Armor systems have advanced with the improvement of the metallic, ceramic and lightweight composite materials used, and the both active and passive dynamic protections development have created effective systems against the modern ammunition [4]

The symmetry of the cumulative jet formation process is crucial for the jet penetrating ability, which is of particular importance for special purpose charges that form a jet with predefined characteristics. Therefore, one of the tasks of this paper is to determine the most important causes that lead to deviations from the symmetry. A physical-mathematical model is proposed to describe the influence of the deviations in the symmetry of the cumulative liner, namely the unequal thickness of its wall, on the symmetry of the cumulative jet formation process. Using numerical simulation, a quantitative assessment of this effect is made on the basis of the model, for different meaning of wall thickness variations at different thicknesses of the cumulative liner wall.

\section{Factors Influencing the Symmetry of the Cumulative Jet Forma- TION PROCESS}

The shaped charges are explosive charges with a cavity at one end and a detonator at the other (Fig. 1). The cavity can take the form of a cone, hemisphere, or tulip and is usually lined with a metal insert of copper, steel, aluminum, etc. After a detonation pulse has been applied to the detonator, a detonation wave occurs and propagates along the charge axis. This wave, moving towards the tip of the cone generating line, causes a dynamic deformation of the liner walls in the direction of the charge symmetry axis, whereby the pressure in the material drastically increases. The pressure of the detonation products reaches values far exceeding the yield point of the material from which the cumulative liner is made, so the motion of the latter under the action of the explosion is similar to that of a liquid, however it is caused not by melting but by plastic deformation. Modern designs also include a screen - an inert body that changes the direction of the detonation wave front.

The parameters and characteristics (and ultimately the penetration abilities) of the cumulative jet are highly dependent on the symmetry of the process of its formation 


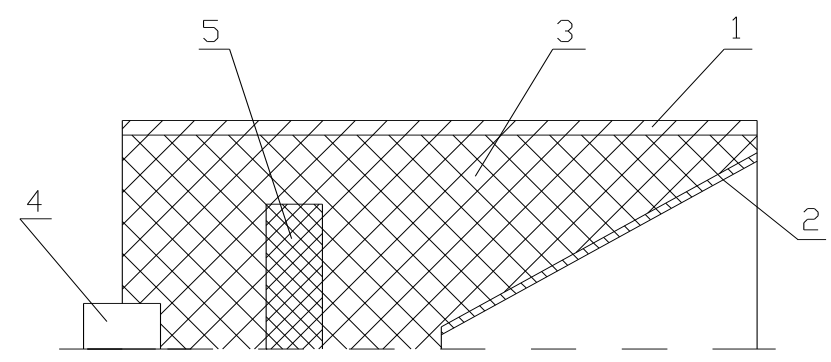

Fig. 1: Principle scheme of a shaped charge: 1 - casing, 2 - liner, 3 - explosive charge, 4 - detonator, 5 - screen.

due to the overall impulse contraction of the cumulative liner, which is theoretically an axisymmetric phenomenon. It is known that during its movement, the cumulative jet is extended as a result of the different speeds along its length. The jet particles have to reach the bottom of the crater created by the preceding particles in the target material without interacting with the crater walls, in order to expend all kinetic energy for increasing the penetration depth. Even a slight deviation from the axis results in such interaction leading to a decrease in penetration efficiency. However, achieving perfect jet symmetry is practically impossible. Any defect or inhomogeneity in the explosive charge, deviation from the dimensions of the liner and the casing, inaccuracy in their assembly or an imperfection of the initiating system could damage the charge axial symmetry and asymmetry of the resulting jet. Each of these factors induces radial velocities throughout the jet, causing deviations from the axis and a deterioration of the penetration characteristics, depending on the magnitude of these velocities $[5,6]$.

Design and optimization of a cumulative charge with predefined characteristics includes not only the choice of a suitable geometry of its parts, but also a selection of materials, manufacturing methods, assembly and filling technologies, current and final control, etc. [7,8]. This makes these types of charges particularly sensitive to the variations in shape and the individual elements positioning $[5,6,9]$. Through the examination of the influence of structural parameters (cone angle, wall thickness, size deviations and detonation initiation point), in [10] it has been found that the initiation point has the greatest effect on the depth of the jet penetration $(22.61 \%)$, and the wall thickness effect is $11.09 \%$. According to [6], the defects in the cone geometry have greater influence on the symmetry than other imperfections such as non-central initiation of the explosive charge, separation of the explosive filling from the casing and presence of air bubbles in the explosive substance volume.

It should be emphasized, as noted by the authors of [10], that the determining 
factor for the degree of the jet deviation is not the type of asymmetry/defect, but its location and magnitude. Thus the results of the studies should not be accepted as criteria for determining what type of asymmetry may be allowed in the charge configuration. Rather, potential defects must be taken into account when seeking a reasonable balance between the individual design parameters in order to achieve the specified penetration capacity of the formed cumulative jet.

Considering that in addition to asymmetries, other factors (such as the physicalmechanical characteristics of materials, temperature, etc., which have been subject of a number of studies [11-21]), also play a vital role, their optimal combination represents a complex and a labor-intensive process. For example, narrowing the tolerances in the manufacture of individual parts or increasing the accuracy during installation does not mean that the armor penetration will increase. This is only possible with a comprehensive consideration of all factors at the stages of research, development, production, and control, including feedback - analysis of test results or their practical use.

For the purpose of this study, an arbitrary cross section of a shaped charge is considered, with outer and inner generating lines of the casing - respectively $\xi=$ $\xi(z)$ and $\eta=\eta(z)$, with outer and inner generating lines of the liner - respectively $\varphi=\varphi(z)$ and $r=r(z)$ (Fig. 2).

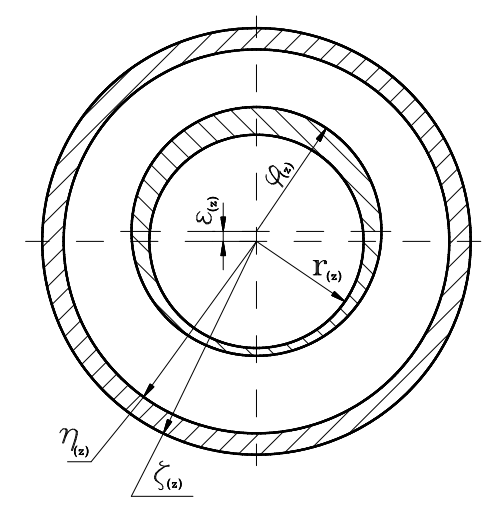

Fig. 2: Cross-sectional diagram of the casing and cumulative liner with varying wall thickness.

Non-simultaneous reaching the charge symmetry axis by the two diametrically positioned elements of the liner may be a result of their asymmetrical initial location, different speeds of movement towards the axis, or the interaction of these two factors. The possible reasons for this may be: variations in the thickness of the liner wall; mutual radial beating of the liner and the casing; variations in the explosive substance 
density; the detonation front non-simultaneous movement towards the external generating line of the liner (axial and radial beating of the screen; misalignment of the point of initiation - the detonator), etc. All these imperfections contribute in varying degrees to the displacement of the symmetry axis of an elementary section of the cumulative jet with respect to the symmetry axis of the charge and therefore lead to the appearance of a lateral pulse, thus dispersing the jet.

One of the common defects is the change in the thickness $\delta$ of the cumulative liner, i.e. $\delta=\delta()$, and the special case is the displacement of the center of the outer generating line $\varphi$ against that of the liner inner generating line $r_{i}$ with magnitude $\xi_{i}$ where $(I=1, \ldots, N)$ is the number of layers into which the cumulative charge is divided following the procedure described in $[11,13]$.

Using the hypothesis that there is no axial flow of the material at its radial flow, according to the law of storage of volumes, the geometrical ratios for the current thickness of the housing $\delta_{1}$ and the liner $\delta_{2}$ in the layer under consideration are as follows:

$$
\begin{aligned}
& \delta_{1}(z, t)=\sqrt{\eta^{2}(z, t)+2 \eta\left(z, t_{0}\right) \delta_{1}\left(z, t_{0}\right)+\delta_{1}^{2}\left(z, t_{0}\right)}-\eta(z t), \\
& \delta_{2}(z, t)=\sqrt{r^{2}(z, t)+2 r\left(z, t_{0}\right) \delta_{2}\left(z, t_{0}\right)+\delta_{2}^{2}\left(z, t_{0}\right)}-r(z, t) .
\end{aligned}
$$

It is obvious that at the overall impulse contraction of the liner, the two diametrically arranged elements (Fig. 2) will travel differently at different speeds until their mutual impact at a certain distance $y$ from the axis of symmetry of the shaped charge. Therefore,

$$
y(z)=y[\varepsilon(z, o),(z, o)],
$$

where $(z, o)$ is a function that takes into account all other physical-mechanical and geometrical parameters and characteristics that initially form certain shaped charge

$$
\sigma(\varepsilon, \dot{\varepsilon})=f(\varepsilon)+K_{1} \dot{\varepsilon}^{K_{2}},
$$

where $K_{1}$ and $K_{2}$ are physical constants of the material determined by the processing of experimental data using the least squares method.

Thus, the following determining equations for real materials are used for calculating the parameters of the dynamic deformation of the cumulative lining and the expansion of the casing of the cumulative unit:

1. For aluminum:

$$
\begin{gathered}
\sigma(\varepsilon, \dot{\varepsilon})=f(\varepsilon)+2.0 \dot{\varepsilon}^{0.4} \mathrm{MPa} \\
f(\varepsilon)=15200 \varepsilon H(0.005-\varepsilon)+[76+82(\varepsilon-0.005)] H(\varepsilon-0.005) \mathrm{MPa} .
\end{gathered}
$$


2. For copper M1:

$$
\begin{gathered}
\sigma(\varepsilon, \dot{\varepsilon})=f(\varepsilon)+0.0086 \dot{\varepsilon}^{1.05} \mathrm{MPa} \\
f(\varepsilon)=18333 \varepsilon H(0.02-\varepsilon)+[275+341(\varepsilon-0.02)] H(\varepsilon-0.02) \mathrm{MPa} .
\end{gathered}
$$

3. For copper M2:

$$
\begin{gathered}
\sigma(\varepsilon, \dot{\varepsilon})=f(\varepsilon)+0.00958 \dot{\varepsilon}^{0.97} \mathrm{MPa} \\
f(\varepsilon)=14250 \varepsilon H(0.02-\varepsilon)+[285+500(\varepsilon-0.02)] H(\varepsilon-0.02) \mathrm{MPa} .
\end{gathered}
$$

4. For steel C3:

$$
\begin{gathered}
\sigma(\varepsilon, \dot{\varepsilon})=f(\varepsilon)+\left[\sigma_{s}(\dot{\varepsilon})-231\right]+\frac{\partial \sigma}{\partial \varepsilon}(\varepsilon)[f(\varepsilon)-231] \mathrm{MPa}, \\
\sigma_{s}(\dot{\varepsilon})=14.6 \sqrt{\dot{\varepsilon}+0.1}+220 \mathrm{MPa} \\
\frac{\partial \sigma}{\partial \varepsilon}\left(\varepsilon_{p}\right)=-0.0474\left[\sqrt{\dot{\varepsilon_{p}}+0.1}+0.96\right] \mathrm{MPa} \\
f(\varepsilon)=2.07 .10^{5} \varepsilon H(0.001-\varepsilon)+[230+631.6 \sqrt{\varepsilon-0.001}] H(\varepsilon-0.001) \mathrm{MPa} .
\end{gathered}
$$

\section{Numerical Simulation of the EFFeCt of ECCENTRicity on the Cu-} MULATIVE JET FORMATION

Using the developed physical and mathematical model of the process of cumulative jet formation (formula 1) and its numerical realization by the method of $[11,13]$, calculations of the influence of eccentricity on the ordinate of the point of impact between the liner walls at different thicknesses of the cumulative liner $\delta$ for a shaped

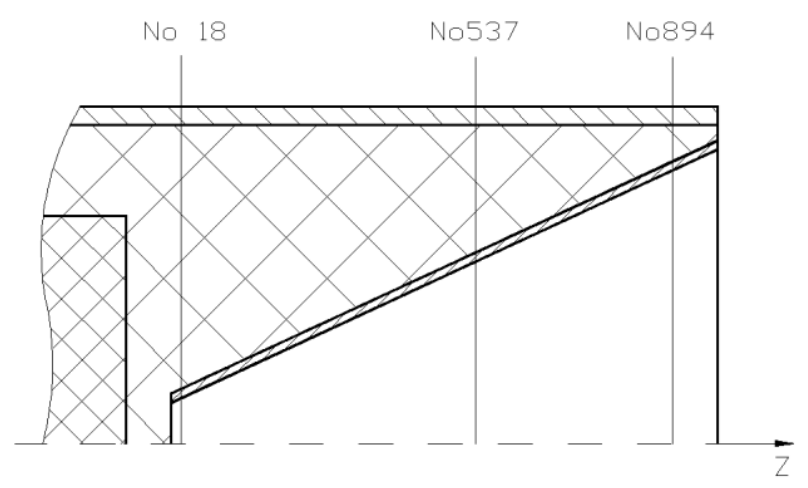

Fig. 3: Diagram of specific layers for which calculations results are presented. 
Table 1: Parameters of the shaped charge under consideration

\begin{tabular}{lc}
\hline \hline Parameter & Value \\
\hline Outer diameter of the casing & $74 \mathrm{~mm}$ \\
Inner diameter of the casing & $70 \mathrm{~mm}$ \\
Liner thickness & $\delta .15$ and $2 \mathrm{~mm}$ \\
Semi-cone angle & $27^{\circ}$ \\
Screen diameter & $50 \mathrm{~mm}$ \\
Distance between the screen and the liner & $5 \mathrm{~mm}$ \\
Casing material & low carbon steel \\
Liner Material & copper 1 \\
Explosive substance -IX-1 & $=1.65 \mathrm{~g} / \mathrm{cm}^{3}$ \\
Detonation velocity & $7100 \mathrm{~m} / \mathrm{s}$ \\
\hline \hline
\end{tabular}

charge with principle construction shown in Fig. 3 and parameters according to Table 1 are performed.

The task is solved for the maximum number of layers $N=1000$. The results for layers No. 18, No. 537 and No. 894 are presented in Fig. 4 and correspond to the characteristic areas of the liner, that are top, middle and base.

The results of the numerical simulation can be summarized as follows:

1. The magnitude of variations in the thickness of the cumulative liner wall influences almost proportionally the displacement of the ordinate of the point of impact between the walls relative to the shaped charge axis of symmetry.

2. The relationship between the variations in the cumulative liner wall thickness and the displacement of the ordinate of the point of impact between the walls for one and the same layer lessen substantially with the increase of the wall thickness.

3. The greatest displacement of the ordinate of the point of collision of the liner walls from the charge axis of symmetry is obtained for the elements of the liner base at thin walls or large eccentricities. 

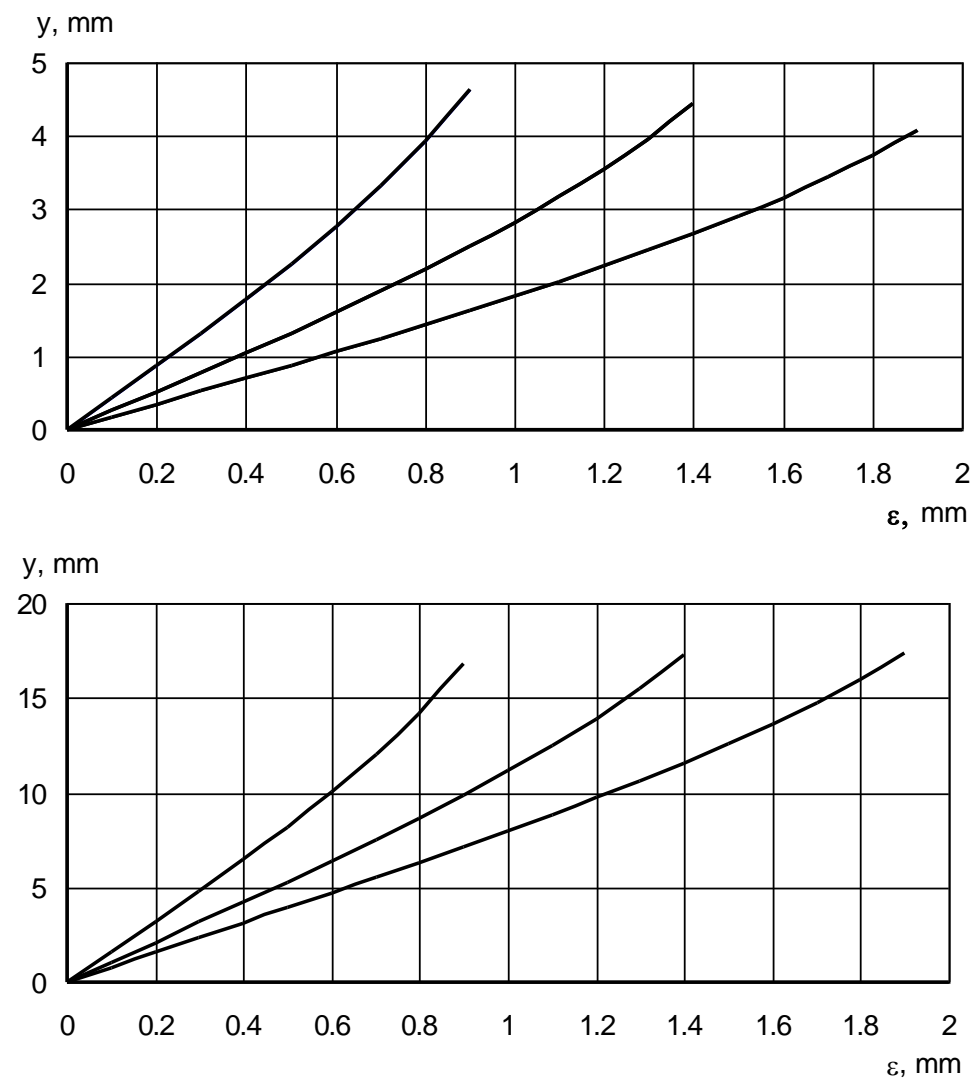

(a) Layer No.18

$\mathrm{y}, \mathrm{mm}$

(b) Layer No.537

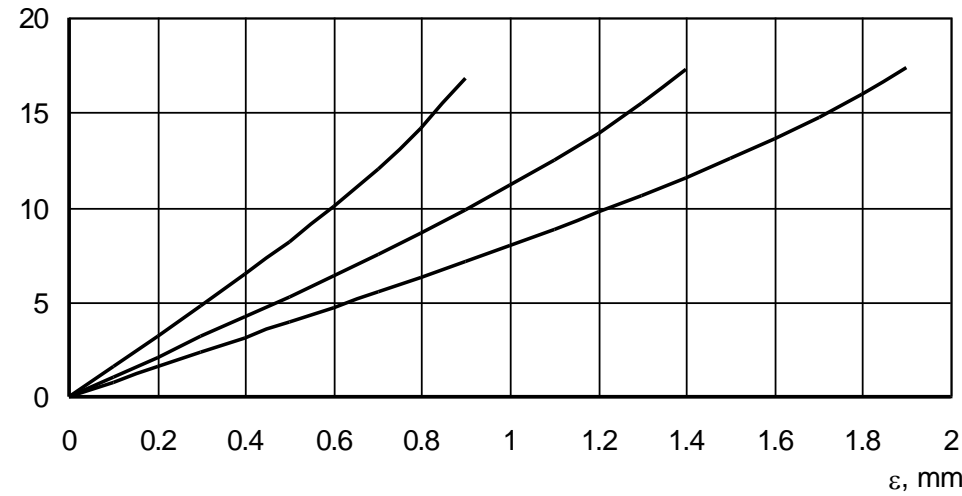

(c) Layer No.894

Fig. 4: Displacement of the ordinate of the point of collision of the liner walls relative to the axis of symmetry for specific layers. 


\section{CONCLUSION}

A physical-mathematical model describing the influence of the deviations in the geometry of the cumulative liner, namely the unequal wall thickness, on the symmetry of the cumulative jet formation process is proposed. The numerical simulation showed that the displacement of the ordinate of the point of collision of the liner walls relative to the axis of charge symmetry increases almost proportionally to the magnitude of variations in thickness and decreases with the increase in thickness of the cumulative liner wall, i.e. the displacement is greatest at thin walls and high eccentricities. This influence is mostly displayed at the base of cumulative liner.

The results obtained could be used in theoretical studies on the cumulative jet functioning, and they could contribute to the optimization of the cumulative charges in the process of their design and manufacturing in order the predefined cumulative jet parameters to be achieved.

\section{REFERENCES}

[1] J.F. Molinari (2002) Finite Element Simulation of Shaped Charges. Finite Elements in Analysis and Design 38(10) 921-936.

[2] W. WAlters (2008) A Brief History of Shaped Charges. Keynote paper. In: Proceedings of the 24th International Symposium on Ballistics, New Orleans, LA, 13-10.

[3] H. SheKhar (2012) Theoretical Modelling of Shaped Charges in the Last Two Decades (1990-2010): A Review. Central European Journal of Energetic Materials 9(2) $155-185$.

[4] B.B. Singh, G. Sukumar, P.P. Senthil, P.K. Jena, P.R. S. Reddy, K.S. KuMAR, V. MADHU, G.M. REDDY (2017) Future Armour Materials and Technologies for Combat Platforms. Defence Science Journal 67(4) 412-419.

[5] W. WALters (2007) Introduction to Shaped Charges. Report No ARL-SR-150, Army Research Laboratory, Aberdeen Proving Ground, USA.

[6] O. AYISIT (2008) The influence of asymmetries in shaped charge performance. International Journal of Impact Engineering 35(12) 1399-1404.

[7] K.K. Andreev, A.F. Beliaev. (1960) "Theory of explosives". Moscow, Oboronprom (in Russian).

[8] T.A.E. Elshenawy (2012) Criteria of Design Improvement of Shaped Charges Used as Oil Well Perforators. PhD Thesis, University of Manchester, School of Mechanical, Aerospace and Civil Engineering.

[9] F.J. Mostert, P.J. Knig, K.D. Werneyer (2001) Predicted and Experimental Results of Shaped Charge Penetration with Liners of Measured Wall Thickness Variation. In: Proceedings of the 19th International Symposium of Ballistics, 7-11 May, Interlaken, Switzerland, 741-747.

[10] J.-H. Ou, , J.-B. OU, Y.-J. JHU (2014) The Design and Analysis for Shaped Charge Liner Using Taguchi Method. Int. Journal of Mechanics 8 53-61. 
[11] F.A. Baum, L.P. Orlenko, K.P. Stanyukovich (1960) "Physics of the Explosives". Moskow, Nauka (in Russian).

[12] V.A. Odintsov, L.A. Chudov, V.V. Selivanov (1975) Expansion of a thick-walled cylindrical shell under the action of an explosive load. News AS USSR, Series "Solid Bodies Mechanics" 5 (in Russian).

[13] V.A. Odintsov, G. Statsenko (1980) Cylinder destruction at the wave stage. News AS USSR, Series "Solid Bodies Mechanics" 2 (in Russian).

[14] V.L. Baranov, K.R. Velikov, I.I. Getsov, S.P. Petkov, P.A. Poltev, Z.C. ChIVIKov (2001) Wave destruction of an elastic-viscoplastic material with initial structural defects. News of the Union of Scientists - Russe, Series "Technical Sciences" 3 57-62 (in Russian).

[15] I.I. Getsov, S.P. Petkov, V.L. Baranov, V.M. Pinaev, S.A. Shvetsov (2001) Continuous initiation of a burst charge as a way to increase efficiency. News of the Tula State University, Series “Problems of special engineering” 3 217-223 (in Russian).

[16] V.L. BARAnOV, I.V. LoPA, I.I. Getsov, S.P. PetKov (2002) Radial-wave expansion of a thick-walled uniform cylindrical shell. News of the Tula State University, Series “Problems of special eng." 4(1) 198-203 (in Russian).

[17] M.S. YAVuZA, O. YILDIRIM, N. SERIN (2013) Numerical and Experimental Investigation of Jet Formation and Particulation in Shaped Charges with Tulip-Like Steel Liners. The 12th Hypervelocity Impact Symposium, Procedia Engineering 58 608-616.

[18] C. WANG, J.X. Ding, H.T. ZhaO (2015) Numerical simulation on jet formation of shaped charge with different liner materials. Defence Science Journal 65 279-286.

[19] S.P. Petkov, V.S. Nikolova (2017) Modeling Dynamic Properties of the Cumulative Coating Material and Shell in Cumulative Unit. In: Proceedings of the V International Scientific and Technical Conference "Engineering. Technologies. Education. Security" 1 94-98 (in Bulgarian).

[20] C. WANG, W. XU, T. Li (2017) Experimental and numerical studies on penetration of shaped charge into concrete and pebble layered targets. International Journal of Multiphysics 11(3) 295-313.

[21] J. Yi, Z. WANG, J. Yin, Z. ZhANG (2019) Simulation Study on Expansive Jet Formation Characteristics of Polymer Liner. Materials (Basel) 12(5) 744. 